Pacific Journal of Mathematics

THE APPROXIMATION OF UPPER SEMICONTINUOUS
MULTIFUNCTION BY STEP MULTIFUNCTION 


\title{
THE APPROXIMATION OF UPPER SEMICONTINUOUS MULTIFUNCTIONS BY STEP MULTIFUNCTIONS
}

\author{
Gerald BeER
}

Let $P$ be a right rectangular parallelepiped in $R^{m}$ and let $Y$ be a metric space. If $\Gamma: P \rightarrow Y$ is an upper semicontinuous multifunction such that for each $x$ in $P$ the set $\Gamma(x)$ is nonempty and closed, then there exists a sequence $\left\{\Gamma_{k}\right\}$ of upper semicontinuous closed valued step multifunctions convergent in terms of Hausdorff distance to $\Gamma$ from above. If $\Gamma$ is compact valued and increasing and $P$ is a closed interval, then the convergence can be made uniform. As a consequence of a Dini-type theorem for mutifunctions, the convergence can also be made uniform if $\Gamma$ is compact valued and continuous.

1. Introduction. Let $X$ and $Y$ be topological spaces. A multifunction $\Gamma: X \rightarrow 2^{Y}$ assigns to each $x$ in $X$ a subset $\Gamma(x)$ of $Y$, possibly empty. A multifunction $\Gamma$ is called upper semicontinuous (u.s.c.) at $z$ in $X$ if whenever $V$ is an open subset of $Y$ that contains $\Gamma(z)$ then the set $\{x: \Gamma(x) \subset V\}$ contains a neighborhood of $z$. It is called lower semicontinuous (l.s.c.) at $z$ if whenever an open subset $V$ of $Y$ satisfies $V \cap \Gamma(z) \neq \varnothing$, then $\{x: \Gamma(x) \cap V \neq \varnothing\}$ contains a neighborhood of $z$. It is called continuous at $z$ if $\Gamma$ is both u.s.c. and l.s.c. at $z$, and $\Gamma$ is continuous (resp. u.s.c., l.s.c.) in $X$ if $\Gamma$ is continuous (resp. u.s.c., l.s.c.) at each point of $X$.

The basic theory of semicontinuous multifunctions is presented in Berge [3], Kuratowski [6], and Smithson [9]. We now list a few tangible semicontinuous multifunctions, the first of which is mentioned in [6].

EXAMPLE 1. If $f: Y \rightarrow X$ is onto, then $f^{-1}: X \rightarrow 2^{Y}$ is an u.s.c. (resp. l.s.c.) multifunction if and only if $f$ is a closed (resp. open) single valued function.

ExAmple 2. If $C$ is a closed convex set in $m$-dimensional Euclidean space $R^{m}$, then $\Gamma: C \rightarrow 2^{R^{m}}$ defined by

$$
\Gamma(z)=\{y:\|y\| \leqq 1 \text { and } y \cdot(x-z) \leqq 0 \text { for each } x \text { in } C\}
$$

is an u.s.c. multifunction.

EXAMPLE 3. If $C$ is an arbitrary set in $R^{m}$, then $\Sigma: C \rightarrow 2^{R^{m}}$ defined by 


$$
\Sigma(z)=\{y: \lambda z+(1-\lambda) y \in C \text { for each } \lambda \text { in }[0,1]\}
$$

is called the star multifunction of $C$. Intuitively, the star of $z$ consists of the subset of $C$ that is visible from $z$. If $C$ is a compact (resp. open) set, then $\Sigma$ is an u.s.c. (resp. l.s.c.) multifunction.

The relationship between semicontinuous multifunctions and ordinary real valued semicontinuous functions is as follows: $f: X \rightarrow R$ is u.s.c. (resp. l.s.c.) if and only if $\theta_{f}: X \rightarrow 2^{R}$ defined by $\theta_{f}(x)=$ $(-\infty, x]$ is u.s.c. (resp. l.s.c.). If $f$ is a nonnegative function, then $f$ is u.s.c. (resp. l.s.c.) if and only if $\Gamma_{f}: X \rightarrow 2^{R}$ defined by $\Gamma_{f}(x)=$ $[0, f(x)]$ is u.s.c. (resp. l.s.c.). In the sequel we call $\Gamma_{f}$ the canonical multifunction associated with the nonnegative function $f$.

If $Y$ is a metric space and the values of $\Gamma: X \rightarrow 2^{Y}$ are nonempty closed subsets of $Y$, then there is a different way to view semicontinuity. First, some terminology is required. Let $B_{\varepsilon}[y]$ denote the closed $\varepsilon$-ball about a point $y$ in $Y$. If $C$ is a nonempty closed subset of $Y$, define the $\varepsilon$-parallel body $B_{\varepsilon}[C]$ as the union of balls: $\mathrm{U}_{y \in C} B_{\varepsilon}[y]$. If $C$ and $K$ are nonempty closed subsets of $Y$, then the Hausdorff distance of $C$ from $K$ is

$$
\delta[C, K]=\inf \left\{\varepsilon: B_{\varepsilon}[C] \supset K \text { and } B_{\varepsilon}[K] \supset C\right\} .
$$

This is not a metric only because the distance between two closed sets may be infinite. If we restrict our distance function to the closed and bounded subsets of $Y$, then we do obtain a metric, called the Hausdorff metric. In this context we say that a closed valued multifunction is Hausdorff upper semicontinuous (resp. l.s.c.) at a point $z$ in $X$ if for each positive $\varepsilon$, the set $\left\{x: \Gamma(x) \subset B_{\varepsilon}[\Gamma(z)]\right\}$ (resp. $\left.\left\{x: \Gamma(z) \subset B_{\varepsilon}[\Gamma(x)]\right\}\right)$ contains a neighborbood of $z$. If $\Gamma$ is compact valued, then semicontinuity in the ordinary sense coincides with semicontinuity in this special sense [3]. In general, Housdorff upper semicontinuity is implied by ordinary upper semicontinuity whereas Hausdorff lower semicontinuity implies ordinary lower semicontinuity.

One direction of research in the study of multifunctions involves proving theorems about them that are either analogues or extensions of theorems about single valued functions. Kakutani's extension of the Brouwer fixed point theorem [5] immediately comes to mind, but many other results of interest have been obtained (see, e.g., Ceder [4] and Smithson [10]). In this article we consider the approximation of u.s.c. closed valued multifunctions by u.s.c. step multifunctions.

2. Step multifunctions. Let $P=\left[a_{1}, b_{1}\right] \times\left[a_{2}, b_{2}\right] \times \cdots \times\left[a_{m}, b_{m}\right]$ be a right rectangular parallelepiped in $m$-dimensional Euclidean 
space $R^{m}$. For $j=1,2, \cdots, m$, let $\left\{x_{0}^{(j)}, x_{1}^{(j)}, \cdots, x_{k_{j}}^{(j)}\right\}$ with $x_{0}^{(j)}=a_{j}$ and $x_{k_{j}}^{(j)}=b_{j}$ be a partition of $\left[a_{j}, b_{j}\right]$. The Cartesian product of these $m$ partitions is called a partition of $P$. A partition so described divides $P$ into $\Pi_{j=1}^{m} k_{j}$ subparallelepipeds which we shall call blocks of the partition. For the purposes of integration theory, a step function $f: P \rightarrow R$ is a function that is constant on the interior of each block. We find it convergent to use a more restrictive definition. Given a partition of $P$, define an equivalence relation $\rho$ on $P$ as follows: $x \rho y$ if and only if $x$ and $y$ belong to the same set of blocks. In this article a step function $f$ associated with a particular partition will mean a function that is constant on each equivalence class of $\rho$. Similarly, a step multifunction $\Gamma: P \rightarrow 2^{Y}$ associated with this partition is defined to be a multifunction that is constant on each equivalence class of $\rho$. Here are some simple examples:

EXAMPLE 4. For each partition of a right rectangular parallelepiped $P$ in $R^{m}$, then $\Gamma_{\rho}: P \rightarrow 2^{R^{m}}$ defined by $\Gamma_{\rho}(x)=$ the closure of $x / \rho$ where $x / \rho$ is the equivalence class of $x$ is a compact valued l.s.c. step multifunction.

EXAMPle 5. If $f: P \rightarrow R$ is a nonnegative step function, then the canonical multifunction $\Gamma_{f}$ corresponding to $f$ is a step multifunction.

ExAmple 6. Let $P=[0,1] \times[0,1]$ and let $P^{*}=\{(x, y, 0)$ : $(x, y) \in P\}$. Let $S$ be the union of a finite collection of lines in $R^{3}$ each of which is a translate of a coordinate axis. If $\Sigma$ is the star multifunction of $S \cup P^{*}$ and $h: P \rightarrow P^{*}$ is the natural injection, then $\Sigma \circ h$ is an u.s.c. step multifunction.

THEOREM 1. Let $P$ be a right rectangular parallelepiped in $R^{m}$ and let $\langle Y, d\rangle$ be a metric space. Let $\Gamma: P \rightarrow 2^{Y}$ be a closed valued u.s.c. multifunction such that for each $x, \Gamma(x) \neq \varnothing$. Then there exists a sequence $\left\{\Gamma_{k}\right\}$ of closed valued u.s.c. step multifunctions defined on $P$ such that for each $x$ in $P$ we have

(1) $\Gamma(x) \subset \Gamma_{k+1}(x) \subset \Gamma_{k}(x)$.

(2) $\lim _{k \rightarrow \infty} \delta\left[\Gamma(x), \Gamma_{k}(x)\right]=0$.

Proof. Let $P=\left[a_{1}, b_{1}\right] \times \cdots \times\left[a_{m}, b_{m}\right]$. Our step multifunction $\Gamma_{k}$ will be defined with respect to the partition of $P$ associated with the regular partition of each $\left[a_{j}, b_{j}\right]$ into $2^{k}$ subintervals of equal length. For each block of the partition define $\theta_{B}: P \rightarrow 2^{Y}$ by 


$$
\theta_{B}(z)= \begin{cases}\bigcup_{x \in B} \Gamma(x) & \text { if } z \text { is in } B \\ \varnothing & \text { otherwise } .\end{cases}
$$

Clearly, $\theta_{B}$ is u.s.c.. We now show that $\theta_{B}$ is closed valued, which is to say that $\bigcup_{x \in B} \Gamma_{k}(x)$ is a closed set. To this end let $\left\{y_{n}\right\}$ be a sequence in $\bigcup_{x \in B} \Gamma(x)$ convergent to some point $y$ in $Y$. If infinitely many terms of the sequence belong to the closed set $\Gamma(z)$ for some fixed $z$, then $y$ is in $\Gamma(z)$. Otherwise by passing to a subsequence, the compactness of $B$ allows us to assume that there exists a convergent sequence $\left\{x_{n}\right\}$ of distinct points in $B$ such that $y_{n}$ is in $\Gamma\left(x_{n}\right)$. Now $\{(x, y): x \in B$ and $y \in \Gamma(x)\}$ regarded as a subset of $B \times Y$ is a closed set [8] so that $y \in \Gamma\left(\lim _{n \rightarrow \infty} x_{n}\right)$. This proves that $\bigcup_{x \in B} \Gamma(x)$ is a closed set.

We now define $\Gamma_{k}: P \rightarrow 2^{Y}$ as follows: $\Gamma_{k}(x)=\mathrm{U}_{B} \theta_{B}(x)$. Since u.s.c. multifunctions are closed under finite unions, $\Gamma_{k}$ is u.s.c. for each positive integer $k$. For each $x, \Gamma_{k}(x)$ is a finite union of closed sets and is thus closed. Moreover, since $x$ belongs to at least one block $B_{x}$, we have $\Gamma_{k}(x) \supset \theta_{B_{x}}(x) \supset \Gamma(x)$. Clearly, $\Gamma_{k}$ is a step function, for $\Gamma_{k}\left(x_{1}\right)=\Gamma_{k}\left(x_{2}\right)$ when the points $x_{1}$ and $x_{2}$ belong to the same blocks of the kth partition. Also, for each integer $k$ and each $x$ in $P$ we have $\Gamma_{k+1}(x) \subset \Gamma_{k}(x)$ because each block of the $(k+1)$ st partition that contains $x$ is a subset of a block of the kth partition that contains $x$. Finally, let $\varepsilon>0$ be arbitrary and $z$ be a fixed point of $P$. Since $\Gamma$ is u.s.c. at $z$, there exists a positive $\lambda$ such that

$$
\bigcup\{\Gamma(x):\|x-z\|<\lambda\} \subset B_{\varepsilon}[\Gamma(z)] \text {. }
$$

However, the diameters of the blocks of successive partitions go to zero; so, there exists an integer $n(z)$ such that whenever $k>n(z)$ the diameter of a block in the $k$ th partition will be less than $\lambda$. In particular, if $x$ is a point in a block $B$ of the kth partition that contains $z$, then $\|x-z\|<\lambda$. Hence, if $k>n(z)$, we have

$$
\Gamma_{k}(z)=\bigcup_{B} \theta_{B}(z)=\bigcup_{x \rho_{z}} \Gamma(x) \subset B_{\varepsilon}[\Gamma(x)] .
$$

We observe that the process described in the last theorem can be used to approximate a compact valued u.s.c. multifunction by compact valued u.s.c. step multifunctions, for the union of the values of a compact valued u.s.c. multifunction when restricted to a compact set is compact. Can we always approximate a l.s.c. closed valued multifunction from below by a sequence of l.s.c. step multifunctions? The answer is negative, for consider the compact valued l.s.c. multifunction $\Gamma:[0,1] \rightarrow 2^{R}$ defined by $\Gamma(x)=\{x\}$. Now if $\theta:[0,1] \rightarrow 2^{R}$ is a step multifunction such that $\theta(x) \subset \Gamma(x)$ for each $x$, then $\theta(x)=\varnothing$ for all but finitely many values of $x$. Hence if 
$\left\{\Gamma_{k}\right\}$ is a sequence of step functions that is majorized by $\Gamma$, then

$$
\bigcup_{k=1}^{\infty} \Gamma_{k}(x)=\varnothing
$$

for all but countably many $x$.

We note, however, that if the values of $\Gamma$ are not merely compact convex sets in $R^{n}$ but are moreover compact convex bodies, then a lower approximation can be obtained. As in Theorem 1 our $k$ th multifunction is defined with respect to the partition of $P=$ $\left[a_{1}, b_{1}\right] \times\left[a_{2}, b_{2}\right] \times \cdots \times\left[a_{m}, b_{m}\right]$ associated with the regular partition of each $\left[a_{j}, b_{j}\right]$ into $2^{k}$ subintervals of equal length. For each block $B$ of the $k$ th partition define $\theta_{B}$ as follows:

$$
\theta_{B}(z)= \begin{cases}\bigcap_{x \in B} \Gamma(x) & \text { if } z \text { is in } B \\ R^{m} & \text { otherwise. }\end{cases}
$$

As expected we set $\Gamma_{k}(z)=\bigcap \theta_{B}(x)$. For each $k$ the multifunction $\Gamma_{k}$ is compact convex valued, and for each $x$ we have $\Gamma_{k}(x) \subset \Gamma_{k+1}(x) \subset$ $\Gamma(x)$. Since l.s.c. multifunctions are closed under finite intersections, each $\Gamma_{k}$ is l.s.c.. To establish the convergence fix $z$ in $P$ and let $\varepsilon>0$ be arbitrary. Without loss of generality we can assume that 0 is in the interior of $\Gamma(z)$. Choose $0<\lambda<1$ such that $B_{\varepsilon}[\lambda \Gamma(x)] \supset$ $\Gamma(z)$. Let $\rho$ be the distance between $\lambda \Gamma(z)$ and the complement of the interior of $\Gamma(z)$ and choose $\delta$ so small that $B_{\rho}[\Gamma(x)] \supset \Gamma(z)$ whenever $\|z-x\|<\delta$. There exists an integer $n$ such that when $k>n$ the diameter of a block of the kth partition is less than $\delta$. By the separation theorem if $B_{\rho}[y] \subset \Gamma(z)$, then $\|x-z\|<\delta$ implies that $y$ is in $\Gamma(x)$. It follows that if $x$ is in a block of the $k$ th partition that contains $z$, we have $\lambda \Gamma(z) \subset \Gamma(x)$. Hence,

$$
B_{\varepsilon}\left[\Gamma_{k}(z)\right] \supset B_{\varepsilon}[\lambda \Gamma(z)] \supset \Gamma(z)
$$

and we have shown that $\left\{\Gamma_{k}\right\}$ converges to $\Gamma$ from below in the Hausdorff metric.

Returning to the u.s.c. case the next result shows that a uniform approximation can be obtained if the original multifunction is u.s.c., defined on a closed interval in the line, compact valued, and increasing.

THEOREM 2. Let $\langle Y, d\rangle$ be a metric space and let $\Gamma:[a, b] \rightarrow 2^{Y}$ be a compact valued u.s.c. multifunction such that for each $x$, $\Gamma(x) \neq \varnothing$. Suppose that $\Gamma$ is increasing: if $x_{1} \leqq x_{2}$, then $\Gamma\left(x_{1}\right) \subset$ $\Gamma\left(x_{2}\right)$. Then ithere exists a sequence $\left\{\Gamma_{k}\right\}$ of compact valued u.s.c. step multifunctions convergent uniformly in the Hausdorff metric to $\Gamma$ such that for each integer $k$ and each $x$ in $[a, b]$ we have 
$\Gamma(x) \subset \Gamma_{k+1}(x) \subset \Gamma_{k}(x)$.

Proof. Let $c$ be in $(a, b]$ and let $\left\{c_{n}\right\}$ be an increasing sequence of numbers convergent to $c$. Since the set of all compact subsets of $\Gamma(c)$ is a compact metric space with respect to the Hausdorff metric [7], a subsequence of $\left\{\Gamma\left(c_{n}\right)\right\}$ converges in the Hausdorff metric to a compact subset $K$ of $\Gamma(c)$. Clearly, since $\Gamma$ is increasing, $\lim _{x \rightarrow c^{-}} \Gamma(x)$ exists and equals $K$. Define $g:(a, b] \rightarrow R$ by

$$
g(z)=\delta\left[\Gamma(z), \lim _{x \rightarrow z^{-}} \Gamma(x)\right] .
$$

For each positive integer $k$ we claim that $\{z: g(z) \geqq 1 / k\}$ is a finite set. If not, there exists a sequence $\left\{z_{n}\right\}$ of distinct numbers in $(a, b]$ and points $y_{n}$ in $\Gamma\left(z_{n}\right)$ for each $n$ such that $d\left[y_{n}, \lim _{x \rightarrow z_{n}} \Gamma(x)\right] \geqq 1 / k$. It follows that $d\left[y_{n}, \Gamma(x)\right] \geqq 1 / k$ for each $x<z_{n}$ so that if $n \neq m$, then $d\left[y_{n}, y_{m}\right] \geqq 1 / k$. Evidently, $\left\{y_{n}\right\}$ does not have a convergent subsequence, and this violates the compactness of $\Gamma(b)$. Notice that $\Gamma$ can have only countably many points of discontinuity, for $\Gamma$ is right continuous at each point of $[a, b]$.

Let $\varepsilon$ be a fixed positive number. We first construct an u.s.c. compact valued step multifunction $\theta_{\varepsilon}$ on $[a, b]$ such that $\Gamma(x) \subset \theta_{\varepsilon}(x) \subset$ $B_{\varepsilon}[\Gamma(x)]$ for all $x$. By the above argument we can find a strictly increasing sequence of numbers $a_{1}, a_{2}, \cdots, a_{N-1}$ in $(a, b)$ such that if $a<x<b$ and $x \notin\left\{a_{1}, \cdots, a_{N-1}\right\}$ then $g(x)<\varepsilon$. Set $a_{0}$ equal to $a$ and $a_{N}$ equal to $b$, and define $\Gamma_{i}^{*}$ on $\left[a_{i-1}, a_{i}\right]$ by

$$
\Gamma_{i}^{*}(x)=\left\{\begin{array}{lll}
\Gamma(x) & \text { if } & a_{\imath-1} \leqq x<a_{i} \\
\lim _{x \rightarrow a_{i}} \Gamma(x) & \text { if } & x=a_{i} .
\end{array}\right.
$$

We now claim that $\left[a_{i-1}, a_{i}\right]$ cannot contain a nested sequence of subintervals $\left\{\left[c_{j}, d_{j}\right]\right\}$ such that $d_{j}-c_{j}$ converges to zero and for each $j \delta\left[\Gamma_{i}^{*}\left(c_{j}\right), \Gamma_{i}^{*}\left(d_{j}\right)\right] \geqq \varepsilon$. For suppose that such a nested sequence did exist. Let $p$ be the unique point in each subinterval. Clearly, $p$ can be neither $a_{i-1}$ nor $a_{i}$, for $\Gamma_{i}^{*}$ is right continuous at $a_{i-1}$ and left continuous at $a_{i}$. Hence, $p$ must be between $a_{i-1}$ and $a_{i}$. If $p=c_{j}$ for some index $j$, then $p=c_{k}$ for all $k>j$ and again $\Gamma_{i}^{*}$ would fail to be right continuous at $p$. The remaining possibility is that $p>c_{j}$ for all $j$. A simple argument shows that $g(p) \geqq \varepsilon$ which contradicts $p \notin\left\{a_{0}, a_{1}, \cdots, a_{N}\right\}$. The nonexistence of the nested sequence of subintervals is now verified.

We can now say that for $m_{i}$ sufficiently large, $\left[a_{i-1}, a_{i}\right]$ can be partitioned into $2^{m_{i}}$ subintervals of equal length such that the values of $\Gamma_{i}^{*}$ at adjacent endpoints differ in Hausdorff distance by less than $\varepsilon$. For each $i=1, \cdots, N$, on each of the $2^{m_{i}}$ half-open on the right subintervals of $\left[a_{i-1}, a_{i}\right)$ define $\theta_{\varepsilon}$ to be identically equal to the value 
of $\Gamma_{i}^{*}$ at its right endpoint. Finally, set $\theta_{\varepsilon}(b)$ equal to $\Gamma(b)$.

Using the procedure outlined above we can define compact valued u.s.c. step multifunctions $\Lambda_{1}, \Lambda_{2}, \cdots$, that satisfy $\Gamma(x) \subset \Lambda_{n}(x) \subset$ $B_{1 / n}[\Gamma(x)]$ for each $x$ in $[a, b]$. For each positive integer $k$, let $\Gamma_{k}(x)=\bigcap_{n=1}^{k} A_{n}(x)$. We see that

(1) for each $x \Gamma_{k}(x)$ is compact.

(2) for each $x \Gamma(x) \subset \Gamma_{k+1}(x) \subset \Gamma_{k}(x) \subset B_{1 / k}[\Gamma(x)]$.

(3) $\Gamma_{k}$ is a step multifunction with respect to the partition that is the common refinement of the partitions associated with $\Lambda_{1}, \cdots, \Lambda_{k}$.

The upper semicontinuity of $\Gamma_{k}$ holds because the intersection of an arbitrary family of compact valued u.s.c. multifunctions is u.s.c. This completes the proof.

We now present two counterexamples relevant to the last theorem. The first shows that if $\Gamma:[a, b] \rightarrow 2^{Y}$ is a closed valued increasing u.s.c. multifunction, then it is not always possible to find a uniform approximation even if $\Gamma$ is continuous. Define $\Gamma$ : $[-1,0] \rightarrow 2^{R}$ by

$$
\Gamma(x)= \begin{cases}{[0,-1 / x]} & \text { if }-1 \leqq x<0 \\ {[0, \infty)} & \text { if } x=0\end{cases}
$$

If $\theta$ is a step multifunction that majorizes $\Gamma$, then $\theta$ is constant on an interval of the form $[-\varepsilon, 0)$, and the constant value of $\theta$ on this interval contains $[0, n]$ for each positive integer $n$. Thus, the value of $\theta$ on such an interval includes $[0, \infty)$, and if $x$ is in the interval we have $\delta[\theta(x), \Gamma(x)]=\infty$.

The next example shows that the theorem fails if $\Gamma$ is an increasing u.s.c. compact valued multifunction defined on a rectangle rather than on an interval. In this context $\Gamma$ is called increasing if whenever $x_{1} \leqq x_{2}$ and $y_{1} \leqq y_{2}$ then $\Gamma\left(x_{1}, y_{1}\right) \subset \Gamma\left(x_{2}, y_{2}\right)$. We construct a nonnegative real valued u.s.c. function $f$ on $[0,1] \times[0,1]$ for which $\Gamma_{f}$, the canonical multifunction associated with $f$, serves as a counterexample. Let $f$ be the characteristic function of the closed set $\{(x, y): x+y \geqq 1\}$ restricted to $[0,1] \times[0,1]$. Since $f$ is increasing and u.s.c., so is $\Gamma_{f}:[0,1] \times[0,1] \rightarrow 2^{R}$ defined by $\Gamma_{f}(x, y)=[0, f(x, y)]$. We now show that if $\theta$ is any step multifunction whatsoever that majorizes $\Gamma_{f}$, then there exists $(x, y)$ in the unit square such that $\delta\left[\Gamma_{f}(x, y), \theta(x, y)\right] \geqq 1$. Consider the block of the partition associated with $\theta$ that lies in the upper left-hand corner of the unit square. Since the interior of the block will contain points of the form $(x, 1-x)$ for sufficiently small positive $x$, and $\theta$ is constant on the interior, we conclude that $\theta(x, y) \supset[0,1]$ for each $(x, y)$ in the interior. However, the interior of the block contains points $(x, y)$ that satisfy $x+y<1$. This finishes the argument. 
3. Two consequences of Dini's theorem for multifunctions. If $f$ is a continuous real valued function defined on a right rectangular parallelepiped, then a decreasing sequence of upper semicontinuous step functions that converge to $f$ from above must actually converge uniformly. This follows from Dini's Theorem: Let $\left\{f_{k}\right\}$ be a sequence of u.s.c. real valued functions defined on a compact topological space $X$. Suppose that $f$ is a l.s.c. function defined on $X$ and that $\left\{f_{k}(x)\right\}$ converges monotonically to $f(x)$ from above at each point $x$ in $X$. Then $\left\{f_{k}\right\}$ converges uniformly to $f$. It is easy to extend Dini's Theorem to compact valued multifunctions.

THeOREM 3. Let $X$ be a compact topological space and let $Y$ be a metric space. Suppose that $\Gamma: X \rightarrow 2^{Y}$ is a l.s.c. compact valued multifunction such that for each $x$ in $X, \Gamma(x) \neq \varnothing$. For $k=$ $1,2,3, \cdots$ let $\Gamma_{k}: X \rightarrow 2^{Y}$ be an u.s.c. compact valued multifunction such that for each $x$ in $X$ and each integer $k$ we have $\Gamma_{k}(x) \supset$ $\Gamma_{k+1}(x) \supset \Gamma(x)$. If $\bigcap_{k=1}^{\infty} \Gamma_{k}(x)=\Gamma(x)$ for each $x$, then $\left\{\Gamma_{k}\right\}$ converges to $\Gamma$ uniformly in the Hausdorff metric.

Proof. The compactness of $\Gamma_{k}(x)$ and $\Gamma(x)$ for each $k$ and $x$ imply that $\left\{\Gamma_{k}(x)\right\}$ converges to $\Gamma(x)$ in the Hausdorff metric. Let $\varepsilon>0$ be arbitrary. For each $z$ in $X$ there exists a neighborhood $U_{z}$ of $z$ such that if $x$ is in $U_{z}$, then $\Gamma(z) \subset B_{\varepsilon / 3}[\Gamma(x)]$. Let $n(z)$ be an integer so large that $\Gamma_{n(z)}(z) \subset B_{\varepsilon / 3}[\Gamma(z)]$. Pick $V_{z}$ to be a neighborhood of $z$ such that if $x$ is in $V_{z}$ then $\Gamma_{n(z)}(x) \subset B_{\varepsilon / 3}\left[\Gamma_{n(z)}(z)\right]$. Now let $W_{z}=U_{z} \cap V_{z}$. Since $\left\{W_{z}: z \in X\right\}$ is an open cover of the compact space $X$, we can extract a finite subcover $\left\{W_{z_{1}}, \cdots, W_{z_{q}}\right\}$. If we set $M$ equal to the maximum of $\left\{n\left(z_{1}\right), \cdots, n\left(z_{q}\right)\right\}$, then $k>M$ implies that $\Gamma_{k}(x) \subset B_{\varepsilon}[\Gamma(x)]$ for each $x$ in $X$.

Combining Theorem 1 and Theorem 3 we see that if $\Gamma$ is a compact valued continuous multifunction on a right rectangular parallelepiped, then there exists a sequence of compact valued u.s.c. step multifunctions that converge uniformly to $\Gamma$ from above in the Hausdorff metric. As another application it is easy to show that if the star multifunction $\Sigma$ for a compact set $C$ in $R^{m}$ is continuous, then the sequence of star multifunctions $\left\{\Sigma_{k}\right\}$ for the parallel bodies $\left\{B_{1 / k}[C]\right\}$ when restricted to $C$ converge uniformly to $\Sigma$. Actually, the converse is true. To see this, suppose that $\Sigma$ is not continuous at some point $z$ in $C$. Since $\Sigma$ is u.s.c. at $z$, there exists $\varepsilon>0$ and points $z_{n}$ in $C$ such that $\left\|z_{n}-z\right\|<1 / n$ but $B_{\varepsilon}\left[\Sigma\left(z_{n}\right)\right] \not \supset \Sigma(z)$. Now if $k$ is a positive integer and $n>k$, we see that $\Sigma(z) \subset \Sigma_{k}\left(z_{n}\right)$. Thus, $\delta\left[\Sigma\left(z_{n}\right), \Sigma_{k}\left(z_{n}\right)\right] \geqq \varepsilon$, and $\left\{\Sigma_{k}\right\}$ when restricted to $C$ does not converge uniformly to $\Sigma$. The measure theoretic consequences of this result 
are discussed in [1].

We finally remark that this version of Dini's Theorem does not extend to closed valued multifunctions, even if the approximating multifunctions and limit multifunction are all continuous. What goes wrong, of course, is that the limit multifunction can be l.s.c. without being Hausdorff l.s.c. Again consider the continuous multifunction

$$
\Gamma(x)= \begin{cases}{[0,-1 / x]} & \text { if }-1 \leqq x<0 \\ {[0, \infty)} & \text { if } x=0 .\end{cases}
$$

Let $E$ be the closed set $\{(x, y):-1 \leqq x \leqq 0$ and $y \in \Gamma(x)\}$. We define our sequence $\left\{\Gamma_{k}\right\}$ of continuous closed valued multifunctions that converges to $\Gamma$ (in the sense that $\lim _{k \rightarrow \infty} \delta\left[\Gamma_{k}(x), \Gamma(x)\right]=0$ for all $x$ ) from above in terms of the $1 / k$ parallel bodies of $E$ :

$$
\Gamma_{k}(x)=\left\{y:(x, y) \in B_{1 / k}[E]\right\} \quad(-1 \leqq x \leqq 0) .
$$

We leave it to the reader to check that $\left\{\Gamma_{k}(x)\right\}$ converges to $\Gamma(x)$ for each $x$, but that the convergence is not uniform.

\section{REFERENCES}

1. G. Beer, Continuity properties of the visibility function, Michigan Math. J., 20 (1973), 297-302.

2. Recession cones of nonconvex sets and increasing functions, Proc. Amer. Math. Soc., 73 (1979), 228-232.

3. C. Berge, Topological Spaces, Oliver and Boyd, Edinburgh, 1963.

4. J. Ceder, Compactness and semi-continuous carriers, Proc. Amer. Math. Soc., 14 (1963), 991-993.

5, S. Kakutani, A generalization of Brouwer's fixed point theorem, Duke Math. J., 8. (1941), 457-459.

6. K. Kuratowski, Topology, Academic Press,, New York, 1966.

7. E. Michael, Topologies on spaces of subsets, Trans. Amer. Math. Soc., 71 (1951), 152-182.

8. H. Nikaido, Convex Structures and Economic Theory, Academic Press, New York, 1968.

9. R. Smithson, Multifunctions, Nieuw Arch. Wisk., 20 (1972), 31-53.

10. Some general properties of multivalued functions, Pacific J. Math., 15 (1965), 681-703.

Received July 14, 1978.

California State University

Los ANgeles, CA 90032 



\section{PACIFIC JOURNAL OF MATHEMATICS}

\section{EDITORS}

DONALD BABBITT (Managing Editor)

University of Galifornia

Los Angeles, California 90024

HUGo RossI

University of Utah

Salt Lake City, UT 84112

C. C. MOORE AND ANDREW OGG

University of California

Berkeley, CA 94720

\section{J. DUGUNDJI}

Department of Mathematics University of Southern California Los Angeles, California 90007

R. FINN AND J. MILGRAM Stanford University Stanford, California 94305

ASSOCIATE EDITORS
E. F. BECKENBACH
B. H. NeumanN
F. WoLF
K. YosHIDA

\section{SUPPORTING INSTITUTIONS}

UNIVERSITY OF BRITISH COLUMBIA UNIVERSITY OF SOUTHERN CALIFONIA CALIFORNIA INSTITUTE OF TECHNOLOGY UNIVERSITY OF CALIFORNIA MONTANA STATE UNIVERSITY STANFORD UNIVERSITY UNIVERSITY OF HAWAII UNIVERSITY OF TOKYO UNIVERSITY OF NEVADA, RENO UNIVERSITY OF UTAH NEW MEXICO STATE UNIVERSITY WASHINGTON STATE UNIVERSITY OREGON STATE UNIVERSITY UNIVERSITY OF OREGON UNIVERSITY OF WASHINGTON 


\section{Pacific Journal of Mathematics \\ Vol. 87, No. $1 \quad$ January, 1980}

Spiros Argyros, A decomposition of complete Boolean algebras ..........

Gerald A. Beer, The approximation of upper semicontinuous multifunctions

by step multifunctions . . . . ....................

Ehrhard Behrends and Richard Evans, Multiplicity theory for Boolean

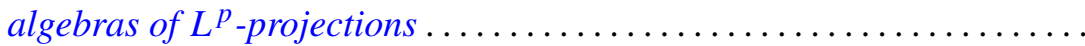

Man-Duen Choi, The full $C^{*}$-algebra of the free group on two

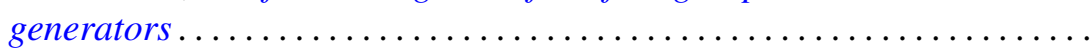

Jen-Chung Chuan, Axioms for closed left ideals in a $C^{*}$-algebra . . . . . . . .

Jo-Ann Deborah Cohen, The strong approximation theorem and locally

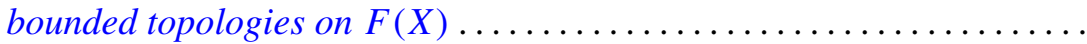

Eugene Harrison Gover and Mark Bernard Ramras, Increasing sequences of Betti numbers............................

Morton Edward Harris, Finite groups having an involution centralizer with

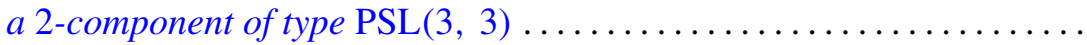

Valéria Botelho de Magalhães Iório, Hopf $C^{*}$-algebras and locally compact

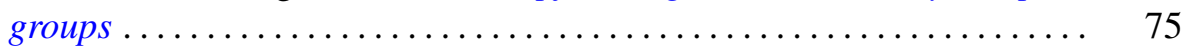

Roy Andrew Johnson, Nearly Borel sets and product measures . . . . . . . . . .

Lowell Edwin Jones, Construction of $Z_{p}$-actions on manifolds . . . . . . . . .

Manuel Lerman and Robert Irving Soare, $d$-simple sets, small sets, and

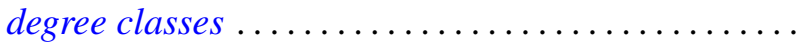

Philip W. McCartney, Neighborly bushes and the Radon-Nikodým property

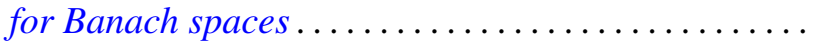

Robert Colman McOwen, Fredholm theory of partial differential equations on complete Riemannian manifolds.

Ernest A. Michael and Carl Preston Pixley, A unified theorem on continuous selections.

Ernest A. Michael, Continuous selections and finite-dimensional sets .

Vassili Nestoridis, Inner functions: noninvariant connected components...

Bun Wong, A maximum principle on Clifford torus and nonexistence of proper holomorphic map from the ball to polydisc.

Steve Wright, Similarity orbits of approximately finite $C^{*}$-algebras . . .

Kenjiro Yanagi, On some fixed point theorems for multivalued

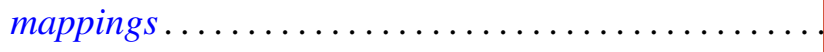

Wieslaw Zelazko, A characterization of LC-nonremovable ideals in commutative Banach algebras 